\title{
Chemical Structure Indices in In Silico Molecular Design
}

\section{Yenamandra S. PrabHAKAR *, Manish K. GUPTA}

Medicinal and Process Chemistry Division, Central Drug Research Institute, Lucknow-226 001, India

\begin{abstract}
The chemical structure indices have a significant role in providing direction to the design of chemotherapeutic agents. These indices come from the experimental domain as well as through the computations. While the experimental properties of compounds have practical value, the indices from computational domain offer fast and economic inputs to simulations. This review article discusses various methods and issues involved in the generation of physicochemical, quantum chemical, graph theoretical, chirality indices, flexible descriptors, molecular fingerprints and 3D chemical space descriptors etc for in silico molecular design approaches. A futuristic perspective of structural indices in drug design is outlined.
\end{abstract}

\section{Keywords}

Chemical structure indices • In silico drug design • QSAR • Molecular modeling

\section{Introduction}

Information encompasses all types of data - qualitative as well as quantitative - which may be processed or unprocessed. It may have been designed to address a simple event or a more complex situation emerged from a statistically defined quantity with a probability of occurrence, etc. In the hierarchy of information flow, notion qualifies as the first qualitative, irregular and vague estimation of information. In this flow, parameters and measurements take the center stage with premonitions (the amorphous state of information) at the origin of the flow line and 
the inferences and decisions at its crystalline end. In it, a parameter is a composition of set of rules designed to define a concept, property or any other relevant information. In the hierarchy of information, a parameter takes precedence over measurement. In this scenario, the origin of the concept of parameterization is time immemorial and it goes along with the consciousness. Figure 1 shows a schematic illustration of hierarchy of information flow which is equally relevant and valid for in silico molecular design approaches.

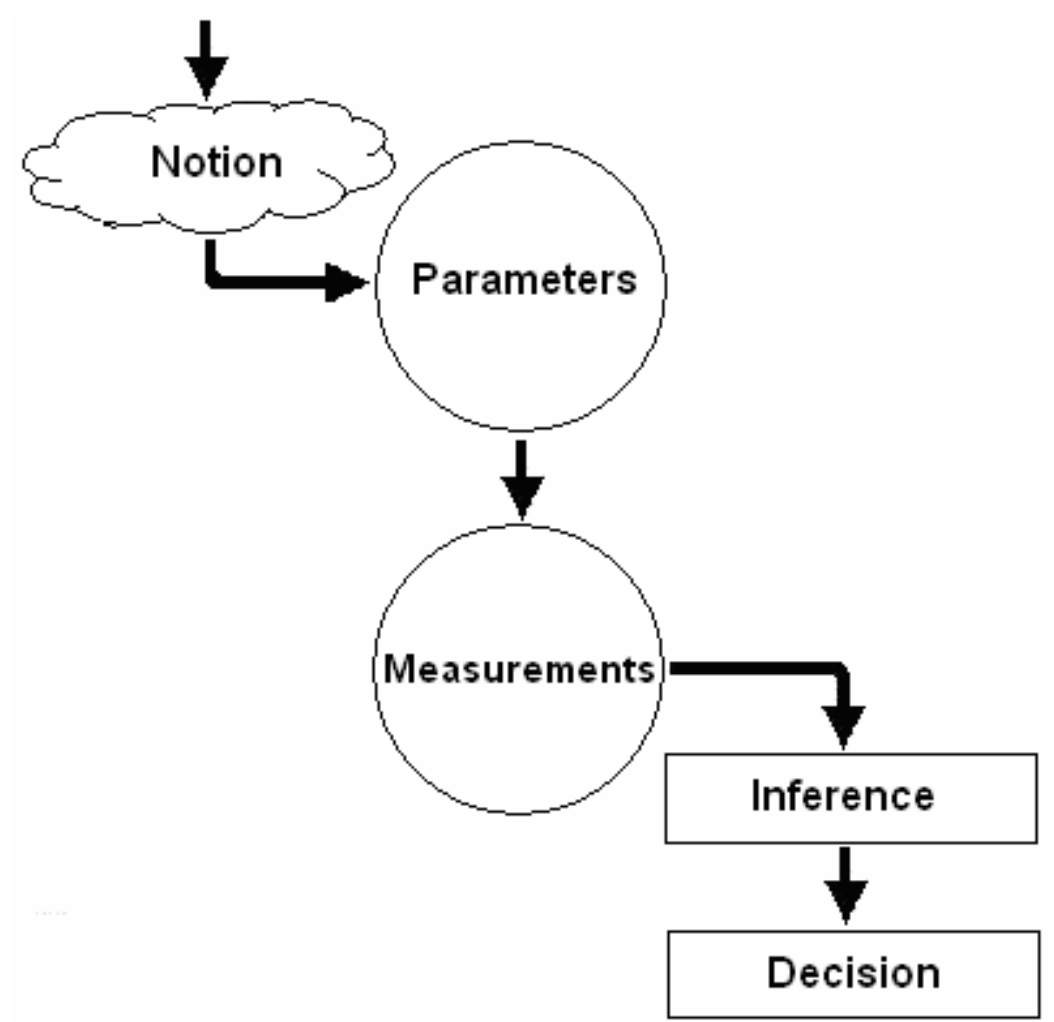

Figure 1. A schematic illustration showing hierarchy of information flow from premonitions to decisions. The irregular enclosure of notion symbolizes its vague nature; the circular enclosures symbolize the ubiquitous as well as universal nature of parameters and measurements; the rectangular enclosures symbolize the welldefined confines of inferences and decisions. In this evolution of 'Notions' to 'Decisions', the chemical structure parameters and their measurements are vital components of in silico molecular design approaches.

A wide spectrum of properties originated from different concepts represents the sequence of chemical information. In this, the compound's nature / atom type etc correspond to one end of the property spectrum and quantum chemical states 
represent the other end of the same. The empirical formulas, molecular formulas, structural formulas etc are spread in between these two extremes. Any physicochemical property or biological activity of a compound is a net result of different forces operating in that compound's environment. This realization has made the whole environment of the compound as a potential source of information to investigate its interplay with the properties and activities. They address different aspects of in silico molecular design and lend credence to the design of additional or new compounds for the chosen goal. The increasing cost of chemical and biochemical studies together with stringent biological and environmental regulations have given impetus to these approaches [1]. Moreover, due to the cost effectiveness, they are in high demand in drug research. The classical and higherdimension graphical quantitative structure activity relationship (QSAR) (also, quantitative structure property relationships i.e. QSPRs) [2,3] procedures and different virtual modeling approaches such as docking [4], virtual screening [5], homology modeling [6] are integral parts of in silico molecular design approaches for drug research. An assumption fundamental and common to all these modeling approaches is that the variation in the biological response can be expressed as a function of the variations in the chemical structure space. These theoretical studies are iterative processes with scope for prediction and feedback tuning for rectification/ improvement, if any. During the last 5 years different reviews have appeared in literature covering the role of topological parameters $[7,8]$, in silico prediction of pharmacokinetic [9] and toxicological profiles [10], drug-drug interactions [11], discrimination of drug like from non-drug like molecules [12], virtual screening methods [5], data-mining techniques [13], feature selection methods [14] etc in drug research. The parameterization chemical structure is an open end challenge. The algorithms and software available today effortlessly compute a few thousand structural indices of compounds for different end uses. In spite of the large number of descriptors, new molecular features are needed to address different aspects so far not tamed, for example, various aspects of molecular recognition [15] challenges of supramolecular chemistry [16] etc. In this 
backdrop, this review article discusses the various methods and issues involved in the generation of different chemical structural indices. A futuristic perspective of structural indices in drug design is outlined.

\section{Chemical Structural Indices}

In isolation, a data point is only a qualified number. A collection of such qualified numbers makes a variable or descriptor. Appropriate parameterization of chemical compounds is essential to comprehend the wealth of information embedded in them. Reliable physicochemical and other property data are also essential to many areas of chemical and biochemical studies which include drugs and pharmaceuticals. Ever since Hansch's [17] and Free and Wilson's [18] observations on the relationship between the chemical structure and its biological response, the progress of classical or 2D-QSAR is largely associated and synonymous with the developments in the numerical characterization of chemical structure and integration of diverse statistical approaches to handle the constantly increasing structural parameters in deriving meaningful inferences. The fundamental QSAR equation of Hansch has been proposed using partition coefficient ( $\log P$ or its derivative $\pi$ ), Hammett $\sigma$ constant as physicochemical descriptors [17]. The Free-Wilson method with indicator variables as descriptors is purely a non-parametric approach [18]. Apart from these physicochemical and indicator variables, several experimental, empirical and non-empirical chemical structural descriptors have been tried in QSAR studies and added to the list of variables as potential descriptors of drug-receptor interactions.

The most common and characteristic numerical descriptors of chemicals are elemental composition, molecular weight, melting point, boiling point, solubility, etc. These parameters represent only a tiny fraction of all possible experimental molecular measurements. The impracticality of experimentally determining physicochemical properties of all existing chemicals can be easily understood. This awareness has generated momentum to find alternative ways of numerical characterization of chemicals. In this context theoretical parameters of chemicals 
which provide reliable estimates of the properties of non-tested as well as not easily amenable compounds are of significant interest.

The descriptors of chemical structures can be broadly categorized as OD (e.g. molecular weight), 1D (e.g., bond/ functional group counts), 2D (structural formula related or $2 \mathrm{D}$ positional descriptors) and $3 \mathrm{D}$ (descriptors from 3D-molecular conformation) parameters. For a classical QSAR study, OD to 2D-structure related parameters or substituent position indices of the compounds form the descriptor database. In these studies, if the compounds of interest belong to a homologous series, then varying structural parts alone are considered for parameterization after excluding the common core structure. The tacit assumption of this practice is that each one of the selected fragments of the whole structure occupies approximately the same position in space with respect to all other analogues. This assumption is true to a very large extent for compounds with rigid structural frames. However, the substituents' relative orientations and location in space are left to the intuitive interpretation of the researchers. In the succeeding parts the molecular descriptors have been addressed according to their origin i.e. physicochemical, quantum chemical, graph theoretical, etc.

\subsection{Physicochemical Indices}

Physicochemical properties are the earliest and most fundamental molecular indices used in drug design approaches. Among them, logP (logarithm of octanolwater partition coefficient) [19, 20], MR (molar refractivity) [19, 20], $E_{s}$ (Taft's steric constant) [21] and $\sigma$ (Hammett sigma constant) [19, 20, 22] are the most common and widely used descriptors in correlation studies. In drug-receptor interactions these descriptors infer the possibilities of hydrophobic/ hydrophilic, polarisability/ dispersive, steric and electronic forces between the ligand and the receptor site. In drug design studies, logP or partition coefficient is one of the most fundamental properties of the compounds responsible not only for interaction at the receptor site but also for its transportation from the site of administration to the site of action. 
Different experimental procedures such as shake-flask method [19], HPLC method [23] etc., are available to determine the logP values of organic compounds. In view of its importance, various computational approaches namely, fragmental constants [19, 20, 24, 25], additive atomic contributions [26, 27], charge densities [28] and conformationally dependent hydrophobic indices [29, 30] are developed to theoretically calculate the logP of a non-ionizable organic compound between two mutually immiscible solvents, 1-octanol and water. Among these computational approaches, fragmental constant methods [19, 20, 24, 25] are the most popular ones. Similar to Hammett $\sigma$ constant, Hansch $\pi$ - constant has been defined to account for the hydrophobic contribution of aromatic substituent groups $\left(\pi_{x}=\log P_{x}-\right.$ $\log P_{H}$, where $P_{X}$ and $P_{H}$ are the partition coefficients of substituted and parent compounds respectively). The subject has been reviewed [19, 31, 32]. Giving due recognition to the $\mathrm{pH}$ of biological system, a $\mathrm{pH}$ based $\log \mathrm{P}$ value $(\log \mathrm{D})$ has been proposed for use in place of logP [33]. Different physicochemical descriptors introduced to model drug-receptor interactions have been shown in Table 1 along with their physical meanings [19-22, 24-26, 33-39].

\subsection{Quantum chemical indices}

Indices from quantum chemical methods provide a precise and complete account of electronic effects of compounds. They offer atom-by-atom as well as cumulative electronic profile of the chemical structure. For a very long time, application of quantum chemical procedures and the indices have been restricted to Huckel Molecular Orbital (HMO) and Extended HMO (EHMO) methods due to the limitations on the part of computational hardware. With the advent of high performance computers, different $a b$ initio and semiempirical quantum-chemical methods have been implemented in theoretical drug design [40, 41]. However, many ab initio quantum chemical calculations are still not practicable in QSAR and modeling studies in view of the large computational requirements of these 
Tab. 1. Some physicochemical parameters used in QSAR and modeling studies

\begin{tabular}{|c|c|c|c|}
\hline Parameters & Symbol & Inference & Ref. \\
\hline \multicolumn{4}{|l|}{ Hydrophobic } \\
\hline Solubility & $\mathrm{s}$ & solubility & 19 \\
\hline Partition coefficient & $\mathrm{K}_{\mathrm{o} / \mathrm{w}}$ & $\begin{array}{l}\text { hydrophilicity / hydrophobicity of } \\
\text { molecules }\end{array}$ & 19 \\
\hline Overall lipophilicity & $\log P$ & do & 19,20 \\
\hline Calculated logP & ClogP & do & 19,20 \\
\hline Retardation factor & $R_{f}$ & do & 19,20 \\
\hline $\begin{array}{l}\text { Chromatographic } \mathrm{R}_{\mathrm{m}} \\
\text { value }\end{array}$ & $\mathrm{R}_{\mathrm{m}}$ & do & 19,20 \\
\hline Distribution coefficient & $\log \mathrm{D}$ & $\begin{array}{l}\mathrm{pH} \text { dependant measure of } \\
\text { hydrophobicity }\end{array}$ & 33 \\
\hline Hydrophobic constant & $\pi$ & $\begin{array}{l}\text { hydrophobicity of chemical atoms } \\
\text { or groups }\end{array}$ & 19,20 \\
\hline $\begin{array}{l}\text { Hydrophobic fragmental } \\
\text { constant }\end{array}$ & $f$ & $\begin{array}{l}\text { hydrophobicity of chemical atoms } \\
\text { or groups }\end{array}$ & $20,24,25$ \\
\hline \multicolumn{4}{|l|}{ Electronic } \\
\hline $\begin{array}{l}\text { Hammett's sigma } \\
\text { constants }\end{array}$ & $\sigma$ & inductive and mesomeric effect & $19,20,22$ \\
\hline Taft's inductive constants & $\sigma^{*}$ & polar / inductive / resonance effect & 34 \\
\hline Mesomeric effect & $M$ & mesomeric and resonance effect & 19,20 \\
\hline Dipole moment & $\mu$ & polarity & 19 \\
\hline Dielectric constant & D & charge & 19 \\
\hline Ionization constants & $\mathrm{pKa}$ & degree of ionization & 19 \\
\hline Electronic field effect & $\mathrm{F}$ & electronic field effect & 35 \\
\hline Resonance effect & $\mathrm{R}$ & electronic resonance effect & 35 \\
\hline NMR chemical shifts & $\delta$ & electron environment of nucleus & 36 \\
\hline \multicolumn{4}{|l|}{ Polarizability } \\
\hline Molar refractivity & MR & Polarizability parameter & $19,20,26$ \\
\hline Molar volume & MV & Polarizability parameter & 19,20 \\
\hline Parachor & PA & Polarizability parameter & 19,20 \\
\hline \multicolumn{4}{|l|}{ Steric } \\
\hline Molecular weight & MW & bulkiness / steric hindrance & 19,20 \\
\hline Bulk steric parameter & $r_{v}$ & do & 19,20 \\
\hline Taft's steric constant & Es & do & 21 \\
\hline Molecular surface area & MSA & do & 19 \\
\hline Sterimol parameters & $\mathrm{L} ; \mathrm{B}_{1}-\mathrm{B}_{5}$ & do & 37 \\
\hline Charton steric parameter & $\mathrm{u}$ & do & 38 \\
\hline van der Waals volume & $V_{v d w}$ & do & 39 \\
\hline
\end{tabular}


calculations. Instead of ab initio methods, semiempirical quantum-chemical methods are often used for the calculation of many electronic descriptors of molecules and their constituent atoms. The most commonly used descriptor are different kinds of charge densities, atomic and molecular orbital (HOMO, LUMO) energies, $\sigma$ and $\pi$ orbital electron densities, $\sigma$ - and $\pi$-bond orders, free-valences, electrophilic-, nucleophilic- and radical-superdelocalizabilities, atom-atom and molecular polarizabilities, dipole moments and a variety of energy indices. The computation of atom-by-atom property profiles of compounds gives scope to analyze them at angstrom level. The increased number of descriptors (due to atomby-atom property profiles) rapidly escalates the time required for their analysis. MOPAC (Molecular Orbital PACkage) is a general-purpose semiempirical molecular orbital package commonly used in QSAR and modeling studies. Gaussian [42] and GAMESS (General Atomic and Molecular Electronic Structure System) [43] are some other quantum chemistry packages of interest to QSAR and modeling studies. Apart from these, different molecular mechanic approaches are also used in molecular modeling for simulations and conformational analysis [44-46]. Some of these aspects have been reviewed [19, 47].

\subsection{Graph Theoretical Indices}

Graph Theoretical Indices are non-empirical descriptors. They account for a very large number of descriptors out of the total used in modeling studies. A graph is an expression of relationship between a set of points (vertices) interconnected through lines (edges). In this perspective all chemical structures are graphical objects with constituent atoms serving as vertices and the chemical bonds between them as edges. They are also addressed as topological graphs of chemical structures. While the topological indices are derived from simple atomic connectivity table of the molecule (2D-structure), Cartesian coordinates of the same are used for deriving the topographical indices. The concepts surrounding this representation of chemical structure in association with the fundamental properties of elements (vertices) have opened avenues for the generation of a very large number of 
characteristic numerical descriptors to quantify the embedded features of chemical structures and parts thereof from different perspectives. Physicochemical properties such as mass, volume, electronegativity or polarizability of vertices are commonly used as weighting factors in the descriptor computation. Almost all topological descriptors are calculated from the $\mathrm{H}$-suppressed molecular graphs. Some of the early and most fundamental graph theoretical descriptors are Wiener index (W), Altenburg polynomial, Gordon and Scantlebury index, Hosoya's Z-index, Randic's connectivity index, Keir and Hall's molecular connectivity indices [48-50].

Several computer programs based on graph theory have been developed to calculate characteristic descriptors of the molecules and their structural fragments. POLLY [51] Molconn-Z [52], TOPS-MODE (TOPological Substructural MOlecular DEsign) [53,54], CODESSA (COmprehensive DEscriptors for Structural and Statistical Analysis) [55, 56], DRAGON [57] and MODEL [58] are some of the well known or recent programs embedded with graph theoretical concepts for characterizing the chemical structure and compute large number of descriptors for modeling and other studies. POLLY uses vertex information content, in terms of properties, in graph theory to computes several topological indices for QSARQSPR analysis [51,59]. The Molconn-Z software computes a wide variety of topological indices addressing molecular connectivities of different orders, shapes, electrotopological states, topological equivalences etc [52]. Estrada proposed TOPS-MODE descriptors based on the spectral moments of the bonds [53,54]. In the computation of these descriptors, the main diagonal entries of the topological matrix are considered in terms of bond weights representing hydrophobic, polarity, electronic and steric features of molecules. They deal with the physicochemical and electronic properties of different regions of the molecule. CODESSA provides an environment to compute different classes of descriptors i.e. topological, geometrical, electronic, quantum chemical as well as multivariate analysis methods [55]. DRAGON provides a platform for the computation of a variety of 2D- and 3Dgraph theoretical descriptors. They include 2DAUTO (2D-autocorrelation), WHIM (Weighted Holistic Invariant Molecular), BCUT (molecular descriptors for the 
chemical similarity/diversity), RDF (Radial Distribution Function), 3D-MoRSE (3DMolecule Representation of Structures based on Electron diffraction), GETAWAY (GEometry, Topology, and Atom-Weights AssemblY) and several other descriptors including $0 \mathrm{D}$ and $1 \mathrm{D}$ categories [57]. Different computational strategies are involved in different groups of descriptors. These strategies include transformations involving autocorrelation functions (2DAUTO) [60-62], eigenvalues, eigenvectors [63], radial distribution function (RDF) [64], electron diffraction data (3D-MoRSE) [65], concepts of leverage (similar to regression diagnostics) (GETAWAY) [66]. Eigenvalues represent the characteristic roots of polynomials. If the origin of the polynomial is adjacency (or distance) matrix of a graph, the resultant eigenvalues are characteristic to that adjacency (or distance) matrix of the graph and represent intramolecular ordering of vertices. Using these concepts several eigenvalue based topological indices have been developed and tested for their ability in correlating physicochemical properties of compounds [63].

Some geometrical descriptors useful in QSAR studies are 3D-Balaban index (J3D) [67], 3D Petitjean shape index (PJI3) [68] and Gravitational indices [69]. Among these, J3D [67] characterizes geometric distance degrees, PJI3 [68] is the fraction of the difference of geometric diameter and radius to the geometric radius, and Gravitational indices [69] reflect the mass distribution in molecules. The information content of these and many other graph theoretical descriptors have been validated through correlation studies involving different additive-constitutive properties of hydrocarbons [70-72] and biological properties of several classes of compounds [73-76]. An interesting application of graph theory is the explanation and prediction of ${ }^{13} \mathrm{C}-\mathrm{NMR}$ profile of organic compounds [77].

In addition to small molecules, the graph theoretical concepts have been extended to address and advance the understanding of characteristics of nucleic acids and proteins. For example, in nucleic acids graph theory has been used for analyzing the features of RNA and DNA and for calculating the sequencedependent melting stability of duplex DNA oligomers [78]. In proteins, the graph theoretical principles have been used to understand their sequence, structure [79], 
domains [80] and internal flexibilities [81]. Its utility has been demonstrated in the characterization of large proteomic maps as well [82].

\subsection{Graph Reconstruction (Inverse QSAR)}

Concepts of molecule rebuilding from QSAR equations or 'inverse QSAR' have developed in tune with QSAR's main objective of identifying structures with desired activity prior to their synthesis. Most of the developments in inverse QSAR revolve around the graph theoretical concepts. It is associated with the reconstruction of structural fragments from the vertex and edge information of descriptors present in identified QSAR equations [83]. It is a mathematical strategy to predict structures of interest from the descriptors of QSAR equations. It is based on the presumption that if nonempirical structure indices $\mathrm{V} 1, \mathrm{~V} 2, \mathrm{~V} 3$... truly encode the basic elements of molecular structure and are related to the property or activity (P) of the molecule, then for a desired (target) property value $\mathrm{P}_{\mathrm{T}}$, a target molecule can be reconstructed using the structural fragments generated from the appropriate $\mathrm{V} 1_{\mathrm{T}}, \mathrm{V}_{\mathrm{T}}, \mathrm{V}_{\mathrm{T}} \ldots$ values [84]. This process of molecule rebuilding from QSAR equations is also addressed by other names such as graph reconstruction, inverse structure generation, inverse imaging etc [84]. Different algorithms have comeup in inverse QSAR to tackle the reconstruction of molecules from their identified characteristics [85-88]. The utility of these inverse QSAR approaches have been validated by reconstruction of structures of interest in various studies involving biological [84, 88], physicochemical [89] and spectral activities and properties [87]. Vertex prioritization schemes have also been used to develop characteristic molecular indices for QSAR/QSPR studies [84].

\subsection{Chirality Indices}

These indices are relatively new to QSAR and modeling studies. The loss of plane of symmetry in molecules results in optical isomerism or chirality. In organic compounds, for all practical purposes, asymmetric substitution of tetrahedral carbon results in optical isomerism. Except for their response to plane polarized light, optical isomers exhibit the same or even identical physicochemical properties, 
viz., hydrophobicity, solubility, steric, electronic, etc. However, biological macromolecules, for example enzymes, generally exhibit high order of selectivity in their affinity for optical isomers. In view of this, different descriptors have been developed to address the chirality of compounds in QSAR models. Schultz et al. have introduced a chiral factor equal to +1 or -1 for asymmetric center with $\mathrm{R}$ - or $\mathrm{S}$ configuration, respectively, in the computation of topological indices of optical isomers [90]. Julian-Ortiz introduced different chirality-sensitive molecular and charge topological indices to predict the properties of chiral compounds [91]. In another approach, attempts have been made to address the chiral atom in terms of groups and atoms attached to it. For this, computation of a few conceptual parameters, namely, flexibility of rotation, probability of availability, and net detachability, have been defined for the atoms/groups attached to the tetrahedral carbon. They have been investigated in a QSAR study of some 3-hydroxy-3methylglutaryl-coenzyme A reductase inhibitors [92]. Golbraikh et al. have introduced several new chirality descriptors starting from the modified Zagreb group index, connectivity indices and topological charge indices with the use of an additional term called chirality correction to vertex degrees of asymmetric atoms in a molecular graph [93]. They extended this approach to compute topological indices to distinguish between $Z$ and $E$ isomers [94]. Randic has introduced graph theorey based two-dimensional chirality descriptors with possible extension to three-dimensional chirality [95]. Rassat et al. have proposed topological rotational strengths as chirality descriptors for polyhedral graphs [96]. QSAR models of enantioselective compounds have been developed with chirality sensitive molecular descriptors [97].

\subsection{Flexible Descriptors}

The concept of flexible descriptors is a new development in QSAR/ QSPR studies. The molecular descriptors so far discussed are rigid in nature and remain with a fixed value for a specific property of the compound or part of it. Randic's variable vertex-connectivity index is an example of flexible descriptor [98]. The main 
difference between Kier and Hall's valence vertex connectivity index [99] (rigid descriptor) and Randic's variable vertex-connectivity index [98] (flexible descriptor) is that while the former uses fixed vertex weights, the optimized vertex weights are used by the latter. Flexible descriptors are also generated from optimization of correlation weights of local graphs invariants [100], linear combinations of connectivity indices [101] and higher level connectivity terms [102]. SMILES (Simplified Molecular Input Line Entry System) notation has also been used in the generation of these descriptors [103]. Most of these flexible descriptors are size dependent. Different biological activities (DHFR inhibitors [104]; anti-HIV-1agents [105]) and physicochemical properties (partial molar volumes of amino acids [106]; aqueous solubility of purine/ pyrimidines [101]) have been modeled with flexible descriptors.

\subsection{Molecular Fingerprints}

These concepts are relatively new to chemoinformatics and modeling studies. Molecular fingerprints are binary representations of chemical structures. Different methods are introduced by different chemical information system companies in a quest to improve the efficiency of operations in chemical database query system. The Daylight Chemical Information Systems fingerprint is normally referred to as a path-based approach or hash-key fingerprints and "learns" from the structures [107]. Unique subgraphs up to a maximum size eight are identified from chemical structure to represent as fixed length bit string. Typical sizes for Daylight fingerprints are 512 or 1024 bits in length. These fingerprints can be folded to decrease the length and increase the bit density. The fingerprints from Molecular Design Limited (MDL) are referred to as "key" based fingerprints [108]. A "key" is a pre-defined pattern matching of the structure and does not "learn" it from the chemical dataset. The number of keys in MDL is 966 . So a fingerprint of maximum 966-bit length is possible from MDL. No folding of fingerprint occurs with this type. Fingerprints of Barnard Chemical Information Systems $(\mathrm{BCl})$ use both of the Daylight and MDL approaches [109]. In this, similar to a dictionary, the keys for the 
fingerprints are first generated from the set and then implemented in the description. Typically in $\mathrm{BCl}$, the fingerprint bit lengths are of the order of 5,000 bits. More methods have come up to encapsulate the geometric [110] and shape [111] information into the fingerprints. All these approaches have variety of applications in drug research concerning to the characterization of chemical structures, diversity/ similarity analysis, selection and acquisition of compound from libraries and databases [110-114]. Hologram QSAR (HQSAR), a QSAR technique devoid of molecular descriptors, also uses characteristic fragment fingerprints (molecular holograms) as predictive variables of biological activity [115]. Recently, Schwaha and Ecker reviewed similarity principles in ligand-based drug discovery and ADMET profiling [116]. The molecular spectra $-{ }^{1} \mathrm{H}$ NMR, MASS, and IR as well as simulated IR and ${ }^{13} \mathrm{C}$ NMR spectra - of compounds have been used as the 3Ddescriptors for the prediction of biological activities [117]. Shell Research Limited has developed EVA (Eigen VAlues) descriptors as molecular field parameters for the 3D-QSAR/ QSPR modeling studies $[58,118]$. They are computed from the normal modes of molecular moments fundamental to IR and Raman spectra in the vibration frequency range of $1-4000 \mathrm{~cm}^{-1}$. Apart from these, QSAR and modeling studies absorb lots of user defined indicator or dummy variables to account for special or distinct characteristics of molecules. All these indicator variables are specific to a molecular site and confine themselves to a limited domain of the particular study.

\subsection{Chemical Space Indices of 3D QSAR}

These indices provide a more realistic account of the nature of the chemical space surrounding the molecule and surmount the limitations of descriptors from 2D-structure. A reliable conformation of chemical structures is a prerequisite for all 3D-approaches. Depending on way of parameterization of conformational space these procedures are categorized as conformational-alignment dependent and alignment free approaches. Pharmacophore and grid based approaches use the alignment dependent descriptors for the development of models. In pharmacophore 
based approaches, the selected pharmacophore pattern and the spatial locations of the remaining structural fragments serve for the generation of the property database of the compounds under investigation [119]. From these indices, atoms and functional groups relevant to the biological activity are identified to model the activity. In grid-based approaches (eg. Comparative molecular field analysis, CoMFA) the descriptors are referred to as 'fields' [120]. In these approaches, first a rectangular (cuboid) grid is generated from the superimposition pattern of appropriate structural conformations of the compounds in the analysis. The chosen fields around each conformation are determined by placing them successively in the grid and by moving a unit function of the selected property (probe atoms/ groups) around their conformational space [120]. Steric, electrostatic, hydrophobic, hydrogen-bond ( $\mathrm{H}$-bond) donor and acceptor properties are the most commonly used fields in grid based approaches. The descriptors of the alignment free approaches (e.g., Comparative molecular moment analysis CoMMA), are computed from the Cartesian and/ or internal coordinates in association with physicochemical/ electronic properties of the atoms of the molecules [121]. Recently Cramer and Wendt reviewed several issues of 3D-QSAR [122].

\section{Conclusion}

Any robust decision support system draws inferences from respective core disciplines in combination with quantification approaches and mathematical formalisms. The type of quantification protocol to be adopted for a given challenge largely depends on the environment of the experiment. The understanding of the challenge helps in translating it into mathematical terms and putting it for tests, which in turn provide inference for decisions. Chemical structures being a component of drug-receptor system, their indices have a significant role in comprehending and rationalising the information content embedded in the drugreceptor interactions. Fujita has reviewed some success stories of conventional QSAR approaches resulting in commercializable bioactive compounds [123]. 
<smiles>Cc1cc(N2CCCC2)cc(/C=C/c2cccc(C(F)(F)F)c2)n1</smiles>

a<smiles>Cc1nc(N2CCCC2)c2[nH]c(-c3ccccc3)cc2n1</smiles>

b<smiles>Cc1cc(N2CCCC2)c2ccc(-c3cccnc3)cc2n1</smiles>

C<smiles>COc1ccc(C)cc1S(=O)(=O)NCCCNc1ncc(C(=O)c2ccccc2C)s1</smiles>

d

Fig. 2. A potential Neuropeptide Y5 receptor (NPY5R) antagonist (compound d) developed from a virtual screening of hydrophobic and topological similarity features of three seed structures (compounds a-c).

A virtual screening of a large database with a pharmacophore model developed from the hydrophobic and topological similarity of three seed structures (compounds a-c, Figure 2) has paved the way for the discovery of a thiazole derivative (compound d; Figure 2) as potential Neuropeptide Y5 receptor (NPY5R) antagonist [124, 125].

More reports are available in literature highlighting the role of structural indices in the predictive and diagnostic aspects of drug-receptor interactions. The main challenge of in silico molecular design approaches is generation of structural fragments from de facto modeling descriptors. Inverse-QSAR is an attempt in this direction [83-89]. Developments in new metrics are required to address different features of drug-receptor interactions so far not tamed. For example, various aspects of molecular recognition [15], supramolecular chemistry [16], co-factor cooperation in drug identification at the receptor etc need more realistic indices. It is of common knowledge that a small variation in the molecule leads to far reaching changes in its activity, for example uracil is a nucleic acid base whereas 5fluorouracil is anticancer drug [126]. The changes in the biological profiles of these 
compounds are far beyond the incremental changes in the physicochemical features of the respective analogues. This type of 'biological-tunnelling-effects' of the compounds needs investigation to identify and quantify the operating forces. In the current state of knowledge 'tailoring' molecules according to the 'needs' of biological activity is still a dream to come true. The developments in the area of artificial intelligence coupled with the chemical indices of features thus far not tamed will come to aid in this direction. Due to space constrains, only selected articles are cited in the review. Even though the economic and environmental constrains have given initial momentum to in silico molecular design approaches, at present it is required due to its ability to provide intuitive reflections of the complex drug-receptor interactions.

\section{Acknowledgements}

The authors thank Mr. Suman K. Mallik, Scientist, Department of Library Science, CDRI, for critical reading of the manuscript. One of the authors (MKG) gratefully acknowledges the CSIR, New Delhi India for the financial support in the form of Senior Research Fellowship. CDRI Communication No. 6945.

\section{References}

[1] Jorgensen WL.

The many roles of computation in drug discovery.

Science. 2004; 303: 1813-1818.

[doi:10.1126/science.1096361]

[2] Kubinyi $\mathrm{H}$.

QSAR and 3D QSAR in drug design Part 2: applications and problems. Drug Discov Today. 1997; 2: 538-546 and references cited therein. [doi:10.1016/S1359-6446(97)01084-2]

[3] Perkins R, Fang H, Tong W, Welsh WJ.

Quantitative structure-activity relationship methods: perspectives on drug discovery and toxicology.

Environ Toxicol Chem. 2003; 22: 1666-1679.

[doi:10.1897/01-171] 
[4] Kitchen DB, Decornez H, Furr JR, Bajorath J.

Docking and scoring in virtual screening for drug discovery: methods and applications.

Nature Rev Drug Discov. 2004; 3: 935-949.

[doi:10.1038/nrd1549]

[5] Waszkowycz B, Perkins TDJ, Sykes RA, Li J.

Large-scale virtual screening for discovering leads in the postgenomic era. IBM Syst J. 2001; 40: 360-376.

[6] Hillisch A, Pineda LF, Hilgenfeld R.

Utility of homology models in the drug discovery process.

Drug Discov Today. 2004; 9: 659-669.

[doi:10.1016/S1359-6446(04)03196-4]

[7] Gozalbes R, Doucet JP, Derouin F.

Application of topological descriptors in QSAR and drug design: history and new trends.

Curr Drug Targets Infect Disord. 2002; 2: 93-102.

[doi:10.2174/1568005024605909]

[8] Hu Q-N, Liang $Y-Z$, Fang $\mathrm{K}-\mathrm{T}$.

The matrix expression, topological index and atomic attribute of molecular topological structure.

J Data Science. 2003; 1: 361-389.

[9] Yamashita F, Hashida M.

In silico approaches for predicting ADME properties of drugs.

Drug Metab Pharmacokinet. 2004; 19: 327-338.

[doi:10.2133/dmpk.19.327]

[10] Dearden JC.

In silico prediction of drug toxicity.

J Comput Aided Mol Des. 2003; 17: 119-127.

[doi:10.1023/A:1025361621494]

[11] Ekins S, Wrighton SA.

Application of In silico approaches to predicting drug-drug interactions.

J Pharmacol Toxicol Methods. 2001; 45: 65-69.

[doi:10.1016/S1056-8719(01)00119-8]

[12] Muegge I.

Selection criteria for drug-like compounds.

Med Res Rev. 2003; 23: 302-321.

[doi:10.1002/med.10041] 
[13] Weaver DC.

Applying data mining techniques to library design, lead generation and lead optimization.

Curr Opin Chem Biol. 2004; 8: 264-270.

[doi:10.1016/j.cbpa.2004.04.005]

[14] Walters WP, Goldman BB.

Feature selection in quantitative structure-activity relationships.

Curr Opin Drug Discov Devel. 2005; 8: 329-333.

[15] Fattori D.

Molecular recognition: the fragment approach in lead generation.

Drug Discov Today. 2004; 9: 229-238.

[doi:10.1016/S1359-6446(03)03007-1]

[16] Hoeben FJ, Jonkheijm P, Meijer EW, Schenning AP.

About supramolecular assemblies of pi-conjugated systems.

Chem Rev. 2005; 105: 1491-1546.

[doi:10.1021/cr030070z]

[17] Hansch C, Fujita T.

$\rho-\sigma-\pi$ Analysis. A Method for the correlation of biological activity and chemical structure.

J Am Chem Soc. 1964; 86: 1616-1626.

[doi:10.1021/ja01062a035]

[18] Free S M Jr, Wilson JW.

A mathematical approach to structure-activity studies.

J Med Chem. 1964; 7: 395-399.

[doi:10.1021/jm00334a001]

[19] Hansch C, Sammes PG, Taylor JB, Ramsden CA, editors.

In: Comprehensive medicinal chemistry: The rational design, mechanistic study and therapeutic application of chemical compounds.

Volume 4, London: Pergamon Press, 1990.

[20] Hansch C, Leo A.

Substituent constants for correlation analysis in chemistry and biology.

NewYork: Wiley-Interscience, 1979 and references cited therein.

[21] Taft RW.

Polar and steric substituent constants for aliphatic and o-benzoate groups from rates of esterification and hydrolysis of esters.

J Am Chem Soc. 1952; 74: 3120-3128.

[doi:10.1021/ja01132a049] 
[22] Hammett LP.

The effect of structure upon the reactions of organic compounds. Benzene derivatives.

J Am Chem Soc. 1937; 59: 96-103.

[doi:10.1021/ja01280a022]

[23] Minick DJ, Frenz JH, Patrick MA, Brent DA.

A comprehensive method for determining hydrophobicity constants by reversed-phase high-performance liquid chromatography.

J Med Chem. 1988; 31: 1923-1933.

[doi:10.1021/jm00118a010]

[24] Rekker RF.

The hydrophobic fragmental constants: Its derivation and applications- A means of characterizing membrane systems.

New York: Elsevier, 1977.

[25] Rekker RF, De Kort HM.

The hydrophobic fragmental constants; an extension to a 1000 data point set.

Eur J Med Chem-Chim Ther. 1979; 14: 479-488.

[26] Viswanadhan VN, Ghose AK, Revankar GR, Robins RK.

Atomic physicochemical parameters for three-dimensional structure directed quantitative structure-activity relationships. 4. Additional parameters for hydrophobic and dispersive interactions and their application for an automated superposition of certain naturally occurring nucleoside antibiotics. J Chem Inf Comput Sci. 1989; 29:163-172 and references cited therein. [doi:10.1021/ci00063a006]

[27] Kantola A, Villar HO, Loew GH.

Atom based parameterization for a conformationally dependent hydrophobic index.

J Comput Chem. 1991;12: 681-689.

[doi:10.1002/jcc.540120605]

[28] Klopman G, Iroff LD.

Calculation of partition coefficients by the charge density method.

J Comput Chem 1981; 2: 157-160.

[doi:10.1002/jcc.540020204]

[29] Hopfinger AJ, Battershell RD.

Application of SCAP to drug design. 1. Prediction of octanol-water partition coefficients using solvent-dependent conformational analyses.

J Med Chem 1976; 19: 569-573.

[doi:10.1021/jm00227a001] 
[30] Bodor N, Gabanyi Z, Wong C-K.

A new method for the estimation of partition coefficient.

J Am Chem Soc 1989; 111: 3783-3786.

[doi:10.1021/ja00193a003]

[31] Mayer JM, van der Waterbeemd H, Testa B.

A comparison between the hydrophobic fragmental method of Rekker and Leo.

Eur J Med Chem-Chim Ther. 1982; 17: 17-25.

[32] Leo A.

Calculating $\log \mathrm{P}_{\text {oct }}$ from structures.

Chem Rev. 1993; 93: 1281-1306.

[doi:10.1021/cr00020a001]

[33] Tetko IV, Poda GI.

Application of ALOGPS 2.1 to predict logD distribution coefficient for Pfizer proprietary compounds.

J Med Chem 2004; 47: 5601-5604 and references cited therein.

[doi:10.1021/jm049509l]

[34] Taft RW.

The general nature of the proportionality of polar effects of substituent groups in organic chemistry.

J Am Chem Soc 1953; 75: 4231-4238.

[doi:10.1021/ja01113a027]

[35] Swain CG, Lupton EC.

Field and resonance components of substituent effects.

J Am Chem Soc 1968; 90: 4328-4337.

[doi:10.1021/ja01018a024]

[36] Beger RD, Buzatu DA, Wilkes JG, Lay JO Jr.

(13)C NMR quantitative spectrometric data-activity relationship (QSDAR)

models of steroids binding the aromatase enzyme.

J Chem Inf Comput Sci. 2001; 41: 1360-1366.

[doi:10.1021/ci010285e]

[37] Ariens EJ, editor.

In: Drug Design.

Volume 7. NewYork: Acadmic Press, 1976: 165-207.

[38] Charton $\mathrm{M}$.

Steric effects. 7. Additional $\mathrm{V}$ constants.

J Org Chem. 1976; 41: 2217-2220.

[doi:10.1021/jo00874a035]

[39] Gupta SP, Prabhakar YS.

Importance of van der Waals Volume in QSAR studies for Drugs.

J Sci Ind Res. 1985; 44: 189-198. 
[40] Cohen NC, Blaney JM, Humblet C, Gund P, Barry DC.

Molecular modeling software and methods for medicinal chemistry.

J Med Chem. 1990; 33: 883-894.

[doi:10.1021/jm00165a001]

[41] Young DC.

Computational chemistry (online book).

John Wiley \& Sons, Inc. 2002: 19-41; www.interscience.wiley.com. [doi:10.1002/0471220655]

[42] Pople JA, Head-Gordon M, Fox DJ, Raghavachari K, Curtiss LA.

Gaussian-1 theory: a general procedure for prediction of molecular energies. J Chem Phys. 1989; 90: 5622-5629.

[doi:10.1063/1.456415]

[43] Schmidt MW, Baldridge KK, Boatz, JA, Elbert ST, Gordon MS, Jensen JH, Koseki S, Matsunaga N, Nguyen KA, Su S, Windus TL, Dupuis M, Montgomery JA Jr.

The general atomic and molecular electronic structure system.

J Comput Chem. 1993; 14: 1347-1363.

[doi:10.1002/jcc.540141112]

[44] Pearlman DA, Case DA, Caldwell JW, Ross WR, Cheatham TE, DeBolt S, Ferguson D, Seibel G, Kollman P.

AMBER, a computer program for applying molecular mechanics, normal mode analysis, molecular dynamics and free energy calculations to elucidate the structures and energies of molecules.

Comp Phys Commun. 1995; 91: 1-41.

[doi:10.1016/0010-4655(95)00041-D]

[45] Case DA, Cheatham TE III, Darden T, Gohlke H, Luo R, Merz Jr. KM, Onufriev A, Simmerling C, Wang B, Woods RJ.

The Amber biomolecular simulation programs.

J Comput Chem 2005; 26: 1668-1688.

[doi:10.1002/jcc.20290]

[46] Halgren TA.

Merck molecular force field. I. Basis, form, scope, parameterization, and performance of MMFF94.

J Comput Chem. 1996; 17: 490-519 and references cited therein. [doi:10.1002/(SICl)1096-987X(199604)17:5/6<490::AID-JCC1>3.0.CO;2-P]

[47] Karelson M, Lobanov VS, Katritzky AR.

Quantum-chemical descriptors in QSAR/QSPR studies.

Chem Rev. 1996; 96: 1027-1043 and references cited herein. [doi:10.1021/cr950202r] 
[48] Hosoya, $\mathrm{H}$.

A newly proposed quantity characterizing the topological nature of structural isomers of saturated hydrocarbons.

Bull Chem Soc Japan. 1971; 44: 2332-2339.

[49] Randic M.

On characterization of molecular branching.

J Am Chem Soc. 1975; 97: 6609-6015.

[doi:10.1021/ja00856a001]

[50] Kier LB, Hall LH. ‘

Molecular connectivity in chemistry and drug research.

New York: Academic Press, 1976.

[51] Basak SC, Harriss DK, Magnuson VR. POLLY, University of Minnesota: Duluth, MN, 1988.

[52] Molconn-Z, ver. 2.07, eduSoft Lc, a Virginia Corporation, Ashland, VA 23005 USA; www.edusoft-Ic.com.

[53] Estrada E.

Spectral moments of the edge adjacency matrix in molecular graphs. 3.

Molecules containing cycles.

J Chem Inf Comput Sci. 1998; 38: 23-27 and references cited therein. [doi:10.1021/ci970030u]

[54] Estrada E, Gutierrez Y, González H.

Modeling diamagnetic and magnetooptic properties of organic compounds with the TOSS-MODE approach.

J Chem Inf Comput Sci. 2000; 40: 1386-1399.

[doi:10.1021/ci000041e]

[55] Katritzky AR, Lobnov V, Karelson M.

CODESSA (Comprehensive Descriptors for Structural and Statistical

Analysis) 1994, University of Florida, Gainesville, FL.

www.codessa-pro.com.

[56] Katritzky AR, Perumal S, Petrukhin R, Kleinpeter E.

CODESSA-based theoretical QSPR model for hydantoin HPLC-RT lipophilicities.

J Chem Inf Comput Sci. 2001; 41: 569-574.

[doi:10.1021/ci000099t]

[57] DRAGON software by Todeschini R, Consonni V, Mauri A, Pavan M. Milano, Italy. 
[58] Li ZR, Han LY, Xue Y, Yap CW, Li H, Jiang L, Chen YZ.

MODEL - molecular descriptor lab: A web-based server for computing structural and physicochemical features of compounds.

Biotechnol Bioeng. 2007; 97: 389-396.

[doi:10.1002/bit.21214]

[59] Basak SC, Mills D.

Development of quantitative structure-activity relationship models for vapor pressure estimation using computed molecular descriptors.

Arkivoc. 2005; 10: 308-320.

[60] Broto P, Moreau G, Vandycke C.

Molecular structures: perception, autocorrelation descriptors and SAR studies.

Eur J Med Chem-Chim Ther. 1984; 19: 71-78.

[61] Clementi S, Cruciani G, Riganelli D, Valigi R, Costantino G, Baroni M, Wold S.

Autocorrelation as a tool for a congruent description of molecules in 3DQSAR studies.

Pharm Pharmacol Lett. 1993; 3: 5-8.

[62] Wagener M, Sadowski J, Gasteiger J.

Autocorrelation of molecular surface properties for modeling corticosteroid binding globulin and cytosolic Ah receptor activity by neural networks.

J Am Chem Soc. 1995; 117: 7769-7775.

[doi:10.1021/ja00134a023]

[63] Balaban AT, Ciubotariu D, Medeleanu M.

Topological indices and real number vertex invariants based on graph eigenvalues or eigenvectors.

J Chem Inf Comput Sci. 1991; 31: 517-523.

[doi:10.1021/ci00004a014]

[64] Hemmer MC, Steinhauer V, Gasteiger J.

Deriving the 3D structure of organic molecules from their infrared spectra.

Vib Spectrosc. 1999; 19: 151-164.

[doi:10.1016/S0924-2031(99)00014-4]

[65] Schuur JH, Selzer P, Gasteiger J.

The coding of the three-dimensional structure of molecules by molecular transforms and its application to structure-spectra correlations and studies of biological activity.

J Chem Inf Comput Sci. 1996; 36: 334-344.

[doi:10.1021/ci950164c] 
[66] Consonni V, Todeschini R, Pavan M, Gramatica P.

Structure/response correlations and similarity/diversity analysis by

GETAWAY descriptors. 2. Application of the novel 3D molecular descriptors to QSAR/QSPRStudies.

J Chem Inf Comput Sci. 2002; 42: 693-705 and references cited therein. [doi:10.1021/ci0155053]

[67] Mihalic Z, Nikolic S, Trinajstic N.

Comparative study of molecular descriptors derived from the distance matrix. J Chem Inf Comput Sci. 1992; 32: 28-37.

[doi:10.1021/ci00005a005]

[68] Bath PA, Poirrette AR, Willett P, Allen FH.

The extent of the relationship between the graph-theoretical and the geometrical shape coefficients of chemical compounds.

J Chem Inf Comput Sci. 1995; 35: 714-716.

[doi:10.1021/ci00026a007]

[69] Katritzky AR, Mu L, Lobanov VS, Karelson M.

Correlation of boiling points with molecular structure. 1. A training set of 298 diverse organics and a test set of 9 simple inorganics.

J Phys Chem. 1996; 100: 10400-10407.

[doi:10.1021/jp953224q]

[70] Galvez J.

Prediction of molecular volume and surface of alkanes by molecular topology.

J Chem Inf Comput Sci. 2003; 43: 1231-1239.

[doi:10.1021/ci034036x]

[71] Torrens F.

Valence topological charge-transfer indices for dipole moments.

Mol Divers. 2004; 8: 365-370.

[doi:10.1023/B:MODI.0000047508.78271.b1]

[72] Bergstrom CA, Wassvik CM, Norinder U, Luthman K, Artursson P. Global and local computational models for aqueous solubility prediction of drug-like molecules.

J Chem Inf Comput Sci. 2004; 44: 1477-1488.

[doi:10.1021/ci049909h]

[73] Gupta MK, Sagar R, Shaw AK, Prabhakar YS.

CP-MLR directed QSAR studies on the antimycobacterial activity of functionalized alkenols-topological descriptors in modeling the activity.

Bioorg Med Chem. 2005; 13: 343-351.

[doi:10.1016/j.bmc.2004.10.025] 
[74] Prabhakar YS, Gupta MK, Roy N, Venkateswarlu Y.

A high dimensional QSAR study on the aldose reductase inhibitory activity of some flavones: topological descriptors in modeling the activity.

J Chem Inf Model. 2006; 46: 86-92.

[doi:10.1021/ci050060u]

[75] Gonzalez MP, Teran C, Teijeira M.

A topological function based on spectral moments for predicting affinity toward $\mathrm{A}_{3}$ adenosine receptors.

Bioorg Med Chem Lett. 2006; 16: 1291-1296.

[doi:10.1016/j.bmcl.2005.11.063]

[76] Saquib M, Gupta MK, Sagar R, Prabhakar YS, Shaw AK, Kumar R, Maulik PR, Gaikwad AN, Sinha S, Srivastava AK, Chaturvedi V, Srivastava R, Srivastava BS.

C-3 alkyl/ arylalkyl-2,3-dideoxy hex-2-enopyranosides as antitubercular agents: Synthesis, biological evaluation and QSAR study.

J Med Chem. 2007; 50: 2942-2950.

[doi:10.1021/jm070110h]

[77] Panaye A, Doucet JP, Fanpp BT.

Topological approach of carbon-13 NMR spectral simulation: application to fuzzy substructures.

J Chem Inf Comput Sci. 1993; 33: 258-265.

[doi:10.1021/ci00012a012]

[78] Benight AS, Pancoska P, Owczarzy R, Vallone PM, Nesetril J, Riccelli PV. Calculating sequence-dependent melting stability of duplex DNA oligomers and multiplex sequence analysis by graphs.

Method Enzymol. 2001; 340: 165-192.

[doi:10.1016/S0076-6879(01)40422-8]

[79] Freudenberg J, Zimmer R, Hanisch D, Lengauer T.

A hypergraph-based method for unification of existing protein structure- and sequence-families.

In Silico Biology. 2002; 2: 339-349.

[80] Xu Y, Xu D, Gabow HN.

Protein domain decomposition using a graph-theoretic approach.

Bioinformatics. 2000; 16: 1091-1104.

[doi:10.1093/bioinformatics/16.12.1091]

[81] Jacobs DJ, Rader AJ, Kuhn LA, Thorpe MF.

Protein flexibility predictions using graph theory.

Proteins. 2001; 44: 150-165.

[doi:10.1002/prot.1081] 
[82] Milan R.

A graph theoretical characterization of proteomic maps.

Int J Quantum Chem. 2002; 90: 848-858.

[doi:10.1002/qua.10060]

[83] Hall LH, Dailey RS, Kier LB.

Design of molecules from quantitative structure-activity relationship models.

3. Role of higher order path counts: path 3.

J Chem Inf Comput Sci. 1993; 33: 598-603 and references cited therein.

[doi:10.1021/ci00014a012]

[84] Klopman G, Raychaudhury C.

Vartex indices of molecular graphs in structure-activity relationships: A study of the convulsant-anticonvulsant activity of barbiturates and the

carcinogenicity of unsubstituted aromatic hydrocarbons.

J Chem Inf Comput Sci. 1990; 30: 12-19.

[doi:10.1021/ci00065a004]

[85] Gordeeva EV, Molchanova MS, Zefirov NS.

General methodology and computer program for the exhaustive restoring of chemical structures by molecular connectivity indexes. Solution of the inverse problem in QSAR/QSPR.

Tetrahedron Comput Methodol. 1990; 3: 389-415.

[doi:10.1016/0898-5529(90)90066-H]

[86] Contreras ML, Valdivia R, Rozas R.

Exhaustive generation of organic isomers.1. Acyclic structures.

J Chem Inf Comput Sci. 1992; 32: 323-330.

[doi:10.1021/ci00008a011]

[87] Kvasnicka V, Pospichal J.

Simulated annealing construction of molecular graphs with required properties.

J Chem Inf Comput Sci. 1996; 36: 516-526.

[doi:10.1021/ci9500703]

[88] Churchwell CJ, Rintoul MD, Martin S, Visco Jr. DP, Kotu A, Larson RS, Sillerud LO, Brown DC, Faulon J-L.

The signature molecular descriptor: 3 . Inverse-quantitative structure-activity relationship of ICAM-1 inhibitory peptides.

J Mol Graph Model. 2004; 22: 263-273 and references cited therein. [doi:10.1016/j.jmgm.2003.10.002]

[89] Skvortsova MI, Fedyaev KS, Palyulin VA, Zefirov NS.

Molecular design of chemical compounds with prescribed properties from QSAR models containing the Hosoya index.

Internet Electron J Mol Des. 2003; 2: 70-85 and references cited therein. 
[90] Schultz HP, Schultz EB, Schultz TP.

Topological organic chemistry. 9. Graph theory and molecular topological indices of stereoisomeric organic compounds.

J Chem Inf Comput Sci. 1995; 35: 864-870.

[doi:10.1021/ci00027a011]

[91] Julian-Ortiz JV, Gregorio Alaponta C, Rios-Santamarinaa I, GarciaDomenecha R, Galveza J.

Prediction of properties of chiral compounds by molecular topology.

J Mol Graph Model. 1998; 16: 14-18.

[doi:10.1016/S1093-3263(98)00013-8]

[92] Prabhakar YS.

Analysis of tetrahedral carbon in QSAR sudies. A case study using hydroxy-3-methylglutaryl-coenzyme a reductase inhibitors.

J Chem Inf Comput Sci. 1999; 39: 650-653.

[doi:10.1021/ci980047s]

[93] Golbraikh A, Bonchev D, Tropsha A.

Novel chirality descriptors derived from molecular topology.

J Chem Inf Comput Sci. 2001; 41: 147-158.

[doi:10.1021/ci000082a]

[94] Golbraikh A, Bonchev D, Tropsha A.

Novel ZE-isomerism descriptors derived from molecula topology and their application to QSAR analysis.

J Chem Inf Comput Sci. 2002; 42:769-787.

[doi:10.1021/ci0103469]

[95] Randic M.

Graph theoretical descriptors of two-dimensional chirality with possible extension to three-dimensional chirality.

J Chem Inf Comput Sci. 2001; 41: 639-649.

[doi:10.1021/ci000115m]

[96] Rassat A, Laszlo I, Fowler PW.

Topological rotational strengths as chirality descriptors for fullerenes.

Chemistry. 2003; 9: 644-651.

[doi:10.1002/chem.200390072]

[97] Kovatcheva A, Golbraikh A, Oloff S, Feng J, Zheng W, Tropsha A.

QSAR modeling of datasets with enantioselective compounds using chirality sensitive molecular descriptors.

SAR QSAR Environ Res. 2005; 16: 93-102.

[doi:10.1080/10629360412331319844] 
[98] Randic M.

Novel graph theoretical approach to heteroatoms in quantitative structureactivity relationships.

Chemom Intell Lab Syst. 1991; 10: 213-227.

[doi:10.1016/0169-7439(91)80051-Q]

[99] Kier LB, Hall LH.

Molecular connectivity VII: specific treatment of heteroatoms.

J Pharm Sci. 1976; 65: 1806-1809.

[100] Krenkel G, Castro EA, Toropov AA.

Improved molecular descriptors based on the optimization of correlation weights of local graphs invariants.

Int J Mol Sci. 2001; 2: 57-65.

[101] Pogliani L.

Modeling purines and pyrimidines with the linear combination of connectivity indices-molecular connectivity "LCCl-MC" method.

J Chem Inf Comput Sci. 1996; 36: 1082-1091.

[doi:10.1021/ci960020d]

[102] Pogliani L.

Modeling properties with higher-level molecular connectivity descriptors.

J Chem Inf Comput Sci. 1999; 39: 104-111.

[doi:10.1021/ci980054g]

[103] Vidal D, Thormann M, Pons M.

LINGO, an efficient holographic text based method to calculate biophysical properties and intermolecular similarities.

J Chem Inf Model. 2005; 45: 386-393.

[doi:10.1021/ci0496797]

[104] Toropov AA, Nesterov IV, Nabiev OM.

QSAR modeling of dihydrofolate reductase inhibitory activity by correlation weighting of nearest neighboring codes.

J Mol Struct. (THEOCHEM) 2003; 622: 269-273.

[doi:10.1016/S0166-1280(02)00662-0]

[105] Toropov AA, Toropova AP, Nesterov IV, Nabiev OM.

Comparison of QSAR models of anti-HIV1 potencies based on labeled hydrogen filled graph and graph of atomic orbitals.

J Mol Struct (THEOCHEM). 2003; 640: 175-181.

[doi:10.1016/j.theochem.2003.08.127]

[106] Randic M, Mills D, Basak SC.

On characterization of physical properties of amino acids.

Int J Quant Chem. 2000; 80: 1199-1209.

[doi:10.1002/1097-461X(2000)80:6<1199::AID-QUA6>3.0.CO;2-M] 
[107] Daylight Chemical Information Systems, Inc. Daylight Clustering Manual; www.daylight.com.

[108] Durant JL, Leland BA, Henry DR, Nourse JG.

Reoptimization of MDL keys for use in drug discovery.

J Chem Inf Comput Sci. 2002; 42: 1273-1280.

[doi:10.1021/ci010132r]

[109] Barnard Chemical Information Ltd. 46 Uppergate Road, Sheffield S6 6BX, UK.

[110] Brown N, McKay B, Gasteiger J.

Fingal: A novel approach to geometric fingerprinting and a comparative study of its application to 3D-QSAR modeling.

QSAR Comb Sci. 2005; 24: 480-484.

[doi:10.1002/qsar.200430923]

[111] Haigh JA, Pickup BT, Grant JA, Nicholls A.

Small molecule shape-fingerprints.

J Chem Inf Model. 2005; 45: 673-684.

[doi:10.1021/ci049651v]

[112] Willett P, Barnard JM, Downs GM.

Chemical similarity searching.

J Chem Inf Comput Sci. 1998; 38: 983-996.

[doi:10.1021/ci9800211]

[113] McGregor MJ, Muskal SM.

Pharmacophore fingerprinting. 1. Application to QSAR and focused library design.

J Chem Inf Comput Sci. 1999; 39: 569-574.

[doi:10.1021/ci980159j]

[114] Downs GM, Barnard JM.

Clustering methods and their uses in computational chemistry.

Rev Comput Chem. 2002; 18: 1-40.

[doi:10.1002/0471433519.ch1]

[115] Winkler DA, Burden FR.

Holographic QSAR of benzodiazepines.

Quant Struct-Act Relat. 1998; 17: 224-231.

[doi:10.1002/(SICI)1521-3838(199806)17:03<224::AID-QSAR224>3.0.CO;26]

[116] Schwaha R, Ecker GF.

The similarity principle - new trends and applications in ligand-based drug discovery and admet profiling.

Sci Pharm. 2008; 76: 5-18.

[doi:10.3797/scipharm.0802-05] 
[117] Bursi R, Dao T, van Wijk T, de Gooyer M, Kellenbach E, Verwer P. Comparative spectra analysis (CoSA): spectra as three-dimensional molecular descriptors for the prediction of biological activities.

J Chem Inf Comput Sci. 1999; 39: 861-867.

[doi:10.1021/ci990038z]

[118] Ferguson AM, Heritage T, Jonathon P, Pack SE, Phillips L, Rogan J, Snaith PJ.

EVA: A new theoretically based molecular descriptor for use in QSAR/QSPR analysis.

J Comput Aid Mol Des. 1997; 11: 143-152.

[doi:10.1023/A:1008026308790]

[119] Langer T, Krovat EM.

Chemical feature-based pharmacophores and virtual library screening for discovery of new leads.

Curr Opin Drug Discov Devel. 2003; 6: 370-376.

[120] Cramer RD III, Patterson DE, Bunce JD.

Comparative molecular field analysis (CoMFA). 1. Effect of shape on binding of steroids to carrier proteins.

J Am Chem Soc 1988; 110: 5959-5967.

[doi:10.1021/ja00226a005]

[121] Silverman BD, Platt DE.

CoMMA: Comparative molecular moment analysis. 3D-QSAR without molecular superposition.

J Med Chem. 1996; 39: 2129-2140.

[doi:10.1021/jm950589q]

[122] Cramer RD, Wendt B.

Pushing the boundaries of 3D-QSAR.

J Comput Aided Mol Des. 2007; 21: 23-32.

[doi:10.1007/s10822-006-9100-0]

[123] Fujita $T$.

Recent success stories leading to commercializable bioactive compounds

with the aid of traditional QSAR procedures.

Quant Struct-Act Relat. 1997; 16: 107-112.

[doi:10.1002/qsar.19970160202]

[124] Guba W, Neidhart W, Nettekoven M.

Novel and potent NPY5 receptor antagonists derived from virtual screening and iterative parallel chemistry design.

Bioorg Med Chem Lett. 2005; 15: 1599-1603.

[doi:10.1016/j.bmcl.2005.01.063] 
[125] Nettekoven M, Guba W, Neidhart W, Mattei P, Pflieger P, Roche O, Taylor S. Isomeric thiazole derivatives as ligands for the neuropeptide $Y 5$ receptor. Bioorg Med Chem Lett. 2005; 15: 3446-3449. [doi:10.1016/j.bmcl.2005.05.009]

[126] Foye WO, Lemke TL, Williams DA. Editors.

Structural features and pharmacologic activity. In: Principles of Medicinal Chemistry.

N Delhi: BI Waverly Pvt Ltd, 1995: 25-49. 